\title{
Measurement errors of electromagnetic fields of industrial frequency in urban areas
}

\author{
Mariya Amirkhanyan ${ }^{1, *}$ and Fedor Bryukhan ${ }^{2}$ \\ ${ }^{1}$ NPO Gidrotekhproekt, Oktyabr'skaya str. 55A, 175400 Valday City, Novgorod Region, Russia \\ ${ }^{2}$ Moscow State University of Civil Engineering, Yaroslavskoe sh. 26, 129337 Moscow, Russia
}

\begin{abstract}
The universal development of electrical grid infrastructure of the cities leads to an increase in the level of electromagnetic pollution of residential and especially industrial areas. Concern of the urban population about human exposure to potential danger of negative influence of electromagnetic fields (EMF) causes the need for large-scale EMF research in urban areas. To conduct this type of study, the estimates of measurement errors of intensities of the electrical and magnetic components of the EMF should be performed. The goal of this research is to assess the EMF measurement errors of the industrial frequency in urban areas. The error estimation of EMF measurements generated by power line communication was performed using the section of the territory of Moscow's North East, as an example. The main statistical characteristics of measurement errors were determined. It is found that, when performing 3 series of measuring, the errors reduce by 1.5-3 times. When strong EMF intensities exist, it is recommended to carry out not one but a series of measurements with subsequent averaging of the results in each investigated point of the area.
\end{abstract}

\section{Introduction}

Due to the universal development of the city's electric grid infrastructure, the level of electromagnetic pollution of residential and especially industrial areas is increasing. For this reason, the population of cities is concerned about the possible negative impact of electromagnetic fields (EMF) on people [1, 2]. Thus, the factor of EMF-phobia has acquired significant importance by now. In connection with these circumstances, EMF studies and first and foremost EMF of the industrial frequency $(50 \mathrm{~Hz})$ are of current interest. The primary hazard of EMFs is their intangibility. For this reason the EMF maps of particular parts of cities began to be developed over the last years [3]. At the same time, the issue of the validity of EMF measurement results is still under-explored. This is mainly due to the wide range of measuring means. Obtaining consistent measurement results is necessary for development of environmental safety products of cities against their electromagnetic pollution. The aim of this research is to assess the EMF measurement errors of the industrial frequency in urban areas.

Usually studies of electromagnetic pollution of urban areas are initiated by municipal authorities and public organizations or conducted as part of engineering surveys to justify

* Corresponding author: amirkhanyan.maria@gmail.com 
the construction project in a inhabited areas [4]. In some cases, similar studies are carried out as part of different scientific study.

\section{Methodology}

The result of measuring of the electrical or magnetic EMF component, as well as any other physical value is random. The error reasons of measuring the intensity of the electrical and magnetic components of EMF are [5]:

- instrumental errors;

- power fluctuations transmitted by elements of the electrical grid infrastructure;

- inaccurate selection of coordinates of the points under study;

- features of the area under study;

- change in weather conditions during a series of measurements and other factors.

It should be noted that most of the sources of these errors are not systematic and as consequence of averaging the measurement results, these errors are compensated mutually to a significant extent.

The true value of the measured value always differs from the recorded measurement result. Usually it is considered that the deviation of the result of the individual measurement in the first approximation of some value $x *$ from the true value $x$ is enclosed between $-\sigma$ and $+\sigma$, where $\sigma$ is the standard deviation of $x$. When performing field measurements the EMF average value $x_{m}^{*}$ is considered as a true value of the measured value, determined from the sample of $N$ measurements of a sufficiently large volume. The standard deviation is also replaced by its sampled value $\sigma^{*}$, determined by the same sample of measurements. Since in most cases the statistical distribution of deviations of the measured value $x^{*}$ from its true value is described by a Gaussian law, the inequality $\left|x^{*}-x_{m}^{*}\right| \leq \sigma^{*}$ means that approximately at 68 percent credible level the true value of $x$ is determined with accuracy $\sigma^{*}$. To avoid cumbersome designations, the sign $" * "$ will be omitted further in the text, although implied.

EMF measurements in the present work were carried out using the Octava-110A device with antennas P6-70 and P6-71 designed for measuring the magnetic (P6-70) and electric (P6-71) fields. Terms of use of the device provided for weather conditions, excluding air temperature below $+5{ }^{\circ} \mathrm{C}$ and above $+40{ }^{\circ} \mathrm{C}$, air humidity above $90 \%$ and atmospheric precipitation. A special built-in program $\mathrm{Signal}^{+}$is provided to register measurement results and to transfer the data to a computer.

Estimation of EMF measurement errors near the power line communication (PLC) was carried out by statistical processing of the results of the series of measurements performed at different times.

\section{Results}

EMF measurements were conducted for the purpose of the present work near Amundsen street and Serebriakov passage on the North East of Moscow. During the study, 3 series of 15 simultaneous measurements of electric and magnetic field intensities ( $E$ and $H$ respectively) were carried out at $1.8 \mathrm{~m}$ above ground at distances of 10.0, 5.0 and $2.4 \mathrm{~m}$ from the axis of the PLC. These measurements were carried out with an interval of 10 minutes. The measurement points were selected within the small areas with a minimal incline and not capable of distorting the configuration EMF.

The results of measurements of the values of $E$ and $H$ are presented in Table 1. In last three lines of Table 1 the statistical characteristics $x_{m}, \sigma$ and $w=\sigma / x_{m}$ (coefficient of variation) are placed. 
Table 1. The results of simultaneous measurements of electric and magnetic field intensities $(E$ and $H)$ at different distances from the PLC.

\begin{tabular}{|c|c|c|c|c|c|c|}
\hline \multirow{2}{*}{$\begin{array}{c}\text { No. of } \\
\text { measurement }\end{array}$} & \multicolumn{2}{|c|}{ Cluster $1(10.0 \mathrm{~m})$} & \multicolumn{2}{c|}{ Cluster 2 $(5.0 \mathrm{~m})$} & \multicolumn{2}{c|}{ Cluster 3 $(2.4 \mathrm{~m})$} \\
\cline { 2 - 7 } & $E, \mathrm{~V} / \mathrm{m}$ & $H, \mathrm{~A} / \mathrm{m}$ & $E, \mathrm{~V} / \mathrm{m}$ & $H, \mathrm{~A} / \mathrm{m}$ & $E, \mathrm{~V} / \mathrm{m}$ & $H, \mathrm{~A} / \mathrm{m}$ \\
\hline 1 & 72.7 & 0.02 & 390 & 0.83 & 743 & 1.38 \\
\hline 2 & 92.7 & 0.04 & 363 & 0.67 & 767 & 1.71 \\
\hline 3 & 150 & 0.02 & 398 & 0.92 & 741 & 1.52 \\
\hline 4 & 192 & 0.05 & 336 & 0.95 & 763 & 1.54 \\
\hline 5 & 202 & 0.07 & 403 & 0.65 & 846 & 1.81 \\
\hline 6 & 156 & 0.03 & 401 & 0.70 & 715 & 1.50 \\
\hline 7 & 113 & 0.12 & 402 & 0.65 & 817 & 1.57 \\
\hline 8 & 96.6 & 0.12 & 376 & 0.78 & 782 & 1.57 \\
\hline 9 & 134 & 0.06 & 336 & 0.66 & 748 & 1.59 \\
\hline 10 & 136 & 0.07 & 298 & 0.88 & 815 & 1.56 \\
\hline 11 & 132 & 0.05 & 276 & 0.87 & 866 & 1.80 \\
\hline 12 & 90.7 & 0.03 & 316 & 0.72 & 912 & 1.85 \\
\hline 13 & 120 & 0.15 & 336 & 0.68 & 945 & 1.64 \\
\hline 14 & 105 & 0.11 & 298 & 0.67 & 719 & 1.65 \\
\hline 15 & 110 & 0.13 & 288 & 0.69 & 710 & 1.62 \\
\hline$x_{m}$ & 127 & 0.071 & 348 & 0.75 & 793 & 1.62 \\
\hline$\sigma$ & 35.4 & 0.042 & 44.2 & 0.103 & 70.2 & 0.123 \\
\hline$w$ & 0.279 & 0.590 & 0.126 & 0.136 & 0.089 & 0.076 \\
\hline
\end{tabular}

According to the results obtained, the electric field intensity varies from $0.07 \mathrm{kV} / \mathrm{m}(10$ $\mathrm{m}$ from the axis of the PLC) to $0.95 \mathrm{kV} / \mathrm{m}$ (2.4 m from the PLC axis). Similarly, the intensity of the magnetic field increases for the indicated distances from 0.02 to $1.85 \mathrm{~A} / \mathrm{m}$. For visual clarity the results of simultaneous measurements of $E$ and $H$ also represented in Figure 1.

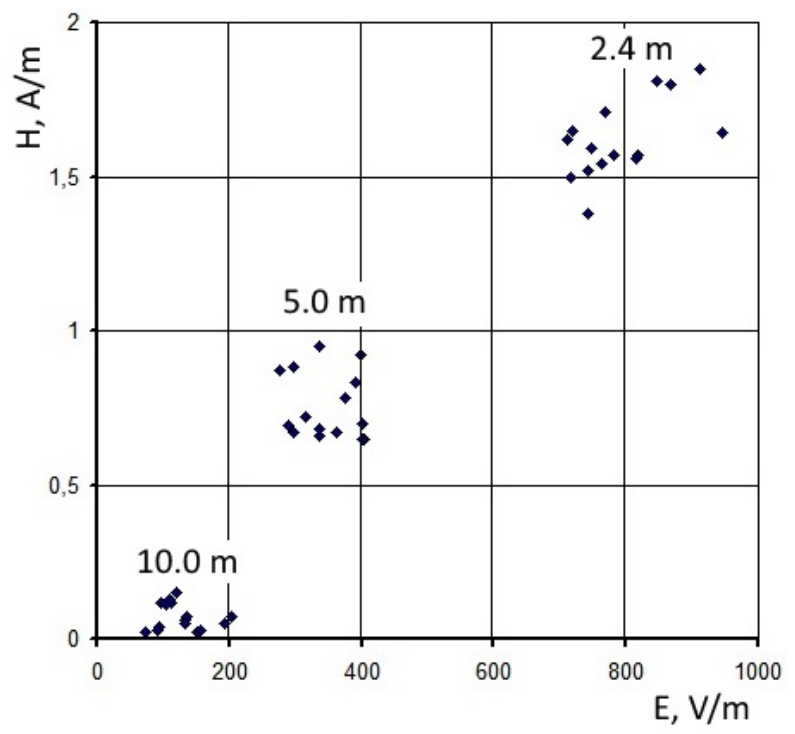

Fig. 1. The results of simultaneous measurements of $E$ and $H$ at different distances from the PLC. 
The coefficient of variation $w$ which is an indicator of the relative measurement error, reaches large values, especially at low EMF intensities. The coefficient of variation is significantly reduced and reaches an acceptable level in the range of medium and moderately strong fields. It should be noted that at the maximum allowable levels of $E$ and $H$ for populated areas which are $5 \mathrm{kV} / \mathrm{m}$ and $16 \mathrm{~A} / \mathrm{m}$ respectively $[6,7]$, the issue of high measurement accuracy in the range of weak and middle fields is not crucial. For strong fields it is possible to recommend to carry out in each investigated point of space not one, but a series of measurements with the subsequent averaging of results.

Figure 1 clearly distinguishes three clusters showing an increase in EMF intensity as it approaches to the axis of the PLC. At the same time, the dispersion of cluster points also increases. The dependency graphs of the variation coefficients of the electric and magnetic components of the EMF from their average values are presented in Figure 2. These graphs clearly show the above-mentioned tendency to reduce the coefficients of variation with EMF intensity increase.
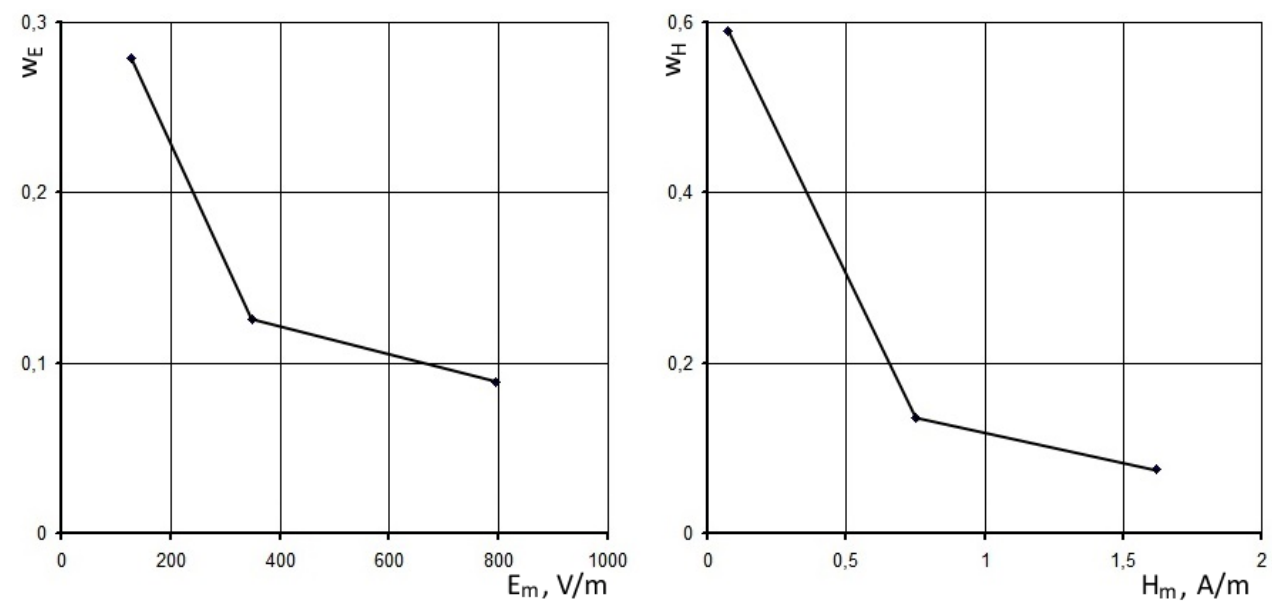

Fig. 2. The dependences of coefficient of variation of electric and magnetic intensity EMF ( $w_{E}$ and $\left.w_{H}\right)$ from their average values $\left(E_{m}\right.$ and $\left.H_{m}\right)$

Usually when performing natural EMF measurements one measurement is carried out at the surveyed points of the area. It is evident that, as the number of measurements increases at a given point and averaging the measurement results, the accuracy of the measurement will also increase. Ther similar recommendation is noted in [5]. An option of selection of 3 measurements wre also investigated in this study. For this purpose, 5 random combinations of 3 measurements were generated out of every 15 measurements in each of the points, the results of which are available in Table 1 . In this case, the values of the measured quantities in each of these 5 combinations were not repeated in other ones. As a true value of the measured quantity, the mean values $x_{m}$ were used from Table 1 . Then the average values for 3 measurements over 5 combinations were determined. The results of the corresponding calculations are presented in Table 2. The standard deviations $\sigma^{\prime}$ are there in the last line of Table 2.

Comparison of the standard deviations presented in Table 2 with the corresponding values from Table 1 shows that the errors are reduced by 1.5-3 times. As previously noted, when strong EMF intensities exist, it is advisable to carry out in each investigated point of the area under study not one but a series of measurements followed by averaging the results. 
It should be noted that the stated approach is also applicable to other means of the field EMF measurements.

Table 2. The results of measurement errors estimate $E$ and $H$ on the basis of 5 sample collections and 3 measurements

\begin{tabular}{|c|c|c|c|c|c|c|}
\hline \multirow{2}{*}{$\begin{array}{c}\text { No. of statistical } \\
\text { sampling }\end{array}$} & \multicolumn{2}{|c|}{$10.0 \mathrm{~m}$} & \multicolumn{2}{c|}{$5.0 \mathrm{~m}$} & \multicolumn{2}{c|}{$2.4 \mathrm{~m}$} \\
\cline { 2 - 7 } & $E, \mathrm{~V} / \mathrm{m}$ & $H, \mathrm{~A} / \mathrm{m}$ & $E, \mathrm{~V} / \mathrm{m}$ & $H, \mathrm{~A} / \mathrm{m}$ & $E, \mathrm{~V} / \mathrm{m}$ & $H, \mathrm{~A} / \mathrm{m}$ \\
\hline 1 & 149 & 0.09 & 329 & 0.74 & 757 & 1.66 \\
\hline 2 & 120 & 0.12 & 323 & 0.67 & 804 & 1.63 \\
\hline 3 & 134 & 0.07 & 360 & 0.74 & 799 & 1.62 \\
\hline 4 & 125 & 0.04 & 338 & 0.78 & 814 & 1.70 \\
\hline 5 & 106 & 0.05 & 388 & 0.84 & 755 & 1.49 \\
\hline$\sigma^{\prime}$ & 14.3 & 0.029 & 23.8 & 0.056 & 24.8 & 0.071 \\
\hline
\end{tabular}

\section{Conclusions}

- Concern among the city population about the potential danger of human exposure to EMF's negative effect makes necessary a large-scale EMF research in urban areas. Similar studies suggest the evaluation of errors in measuring the strengths of the electrical and magnetic components of the EMF.

- Individual measurements of the EMF characteristics contain random errors and it is necessary to perform a series of measurements at fixed points for a reliable determination of these characteristics At the same time, when performing large-scale EMF field studies are usually limited to one measurement.

- The estimation of EMF error measurements of the industrial frequency generated by the PLC in the North East of Moscow was performed. The basic statistical characteristics of errors were determined. It is established that the errors are reduced by 1.5-3 times in the series of 3 measurements. It is noted that it is advisable to carry out in each investigated point of area not one but a series of measurements with subsequent averaging of the results when strong EMF intensities exist.

- The findings of the study allow us to conclude that the proposed technique is also applicable to other means of the field EMF measurements.

\section{References}

1. N.L. Bragazzi, G. Del Puente, Psychol. Res. Behav. Manag., 7, 155-160 (2014)

2. Guidelines for limiting exposure to time-varying electric and magnetic fields $(1 \mathrm{~Hz}$ to 100 kHz), Health Phys., 6, 818-836 (2010)

3. L. Diez, R. Agüero, L. Muñoz, Sensors, 17, 20 (2017)

4. E.P. Polousova, Bull. Moscow State Regional University, ser. "Natural Sciences" 3, 128-129 (2010)

5. Assessment uncertainties relating to electromagnetic fields (EMF) measurement and computation (Health and Safety Executive, Lambourn, Berkshire, 2008)

6. Russian State Standard SanPiN 2971-84

7. Russian State Standard GN 2.1.8/2.2.4.2262-07 